\title{
GROMOV COMPACTNESS FOR HOLOMORPHIC DISCS WITH TOTALLY REAL BOUNDARY CONDITIONS
}

\author{
URS FRAUENFELDER AND KAI ZEHMISCH
}

\begin{abstract}
We prove that a sequence of holomorphic discs with totally real boundary conditions has a subsequence that Gromov converges to a stable holomorphic map of genus zero with connected boundary provided that the sequence is bounded and has bounded energy.
\end{abstract}

\section{INTRODUCTION}

The study of Lagrangian submanifolds in symplectic topology uses holomorphic discs in an essential way. A natural question is the convergence of sequences of parametrized holomorphic discs with boundary values in a given Lagrangian submanifold. By the elliptic nature of the Cauchy-Riemann equation a uniform bound with respect to the $C^{1}$-topology implies convergence of a subsequence including all partial derivatives. A $C^{0}$-bound usually is given by the geometric problem under consideration so that the actual question is about a bound on the gradient.

In general there is no gradient bound. This is because the group of holomorphic automorphisms of the unit disc, which acts on all holomorphic discs by reparametrization, is not compact. On the other hand, the symplectic energy, which is the integral of the symplectic form over the holomorphic disc, is preserved under this action. This implies uniform $L^{2}$-bounds on the gradient provided the holomorphic discs represent the same relative homotopy class. In fact, as observed by Sacks-Uhlenbeck [25] and Gromov [13, locally up to reparametrisations one obtains $C^{\infty}$-convergence using the so-called bubbling off analysis. The limit object consists of finitely many holomorphic discs and spheres glued along finitely many points so that the relative homotopy class is preserved. The so-called Gromov convergence is described in [6] and content of the present article.

Holomorphic discs appear in contact geometry in conjunction with questions about fillability as in $2,13,4,5,8,9,10,11,12,13,14,20,23,27,28$, or the strong Weinstein conjecture [1, 9, 15, 24. One considers contact manifolds that are the boundary of a symplectic manifold. The boundary condition for the holomorphic discs is posed with respect to a totally real submanifold that is not Lagrangian. Because of the integrability of the characteristic distribution in the complement of the singular set uniform gradient bounds along the boundary curve of the holomorphic discs can be obtained by geometric arguments. In order to unify the argumentation and for a deeper understanding of the Reeb dynamics on a contact manifold Gromov convergence of holomorphic discs is required for totally real boundary conditions.

Date: 16.04 .2015$.

2010 Mathematics Subject Classification. 53D12, 53D45, 58J05.

UF partially supported by the Basic Research fund 2013004879 of the Korean government. ZK is partially supported by DFG grant ZE 992/1-1. 
A description how a sequence of holomorphic discs with uniform $W^{1,2}$-bounds and totally real boundary conditions degenerates in the sense of Sacks-Uhlenbeck can be found in [26]. In view of the methods used in symplectic topology a description in the language of stable maps due to Kontsevich [19] as in [6] is required. Approaches that use nodal surfaces similar to Gromov's original description in [13] can be found in [7, 18. An alternative concept makes use of the so-called bubble trees, see 6, 22. To provide an extension of this to holomorphic discs with totally real boundary conditions is the aim of the article.

1.1. Formulation of the theorem. Let $(M, \omega)$ be a symplectic manifold of dimension $2 n$. Let $J$ be an almost complex structure on $M$ that is tamed by $\omega$. We consider a totally real submanifold $L$, which by definition is an $n$-dimensional submanifold $L$ of $M$ such that $T L \cap J T L$ is the zero section. All closed Lagrangian submanifolds and the boundaries in the filling problem are included. A holomorphic disc

$$
u:(\mathbb{D}, \partial \mathbb{D}) \longrightarrow(M, L)
$$

is a smooth map $u: \mathbb{D} \rightarrow M$ defined on the closed unit disc $\mathbb{D} \subset \mathbb{C}$ that is a solution of the Cauchy-Riemann equation

$$
u_{x}+J(u) u_{y}=0,
$$

where $z=x+\mathrm{i} y$ are conformal coordinates on $\mathbb{D}$ and $u_{x}, u_{y}$ stand for $T u\left(\partial_{x}\right)$, $T u\left(\partial_{y}\right)$. We assume that $u$ maps the boundary $\partial \mathbb{D}$ into the totally real submanifold $L$ of $M$.

A sequence $u^{\nu}$ of holomorphic discs is said to be bounded provided that there exists a compact subset of $M$ that contains $u^{\nu}(\mathbb{D})$ for all $\nu \in \mathbb{N}$ and a compact subset of $L$ that contains $u^{\nu}(\partial \mathbb{D})$ for all $\nu \in \mathbb{N}$. We say that $u^{\nu}$ has bounded energy if

$$
\sup _{\nu \in \mathbb{N}} E\left(u^{\nu}\right)<\infty
$$

where the energy of a holomorphic disc $u$ is defined to be

$$
E(u)=\int_{\mathbb{D}} u^{*} \omega
$$

We remark that by the tameness condition the symmetrisation of $\omega(., J$.) is a metric on $M$. With respect to this metric the energy of a holomorphic disc $u$ equals the Dirichlet energy

$$
E(u)=\frac{1}{2} \int_{\mathbb{D}}|\nabla u|^{2}
$$

where the energy density is defined by

$$
|\nabla u|^{2}=\left|u_{x}\right|^{2}+\left|u_{y}\right|^{2}
$$

Theorem 1.1. Any bounded sequence of holomorphic discs with bounded energy has a subsequence that Gromov converges to a stable holomorphic map of genus zero with connected boundary. The relative homotopy class is preserved under Gromov convergence.

The definitions of Gromov convergence and stable maps, which we took from [6], are repeated in Sections [1.3 and 1.5 below. The proof of the theorem is given in Section 3 . The primary idea is to Lagrangefy the boundary condition and is described in Section 2. In Section 4 we will indicate several generalizations. 
1.2. Holomorphic spheres. A holomorphic sphere is a smooth map $u: \mathbb{C} P^{1} \rightarrow$ $M$ that solves the Cauchy-Riemann equation $J \circ T u=T u \circ \mathrm{i}$, where i denotes the complex structure on $\mathbb{C} P^{1}=\mathbb{C} \cup \infty$ obtained by adding the point at infinity to $\mathbb{C}$. The energy of a holomorphic sphere is

$$
E(u)=\int_{\mathbb{C} P^{1}} u^{*} \omega=\frac{1}{2} \int_{\mathbb{C} P^{1}}|T u|^{2},
$$

where the Dirichlet energy integral is taken with respect to the area form induced by the Fubini-Study metric.

1.3. Stable maps. A tree is a finite 1-dimensional simply connected cell complex $T$. The set of 0-cells (or vertices) is denoted by $V$ and the set of 1-cells (or edges) by $E$. An edge is uniquely described by the set of its vertices. To each vertex $\alpha$ we attach a holomorphic map

$$
u_{\alpha}:\left(\Sigma_{\alpha}, \Gamma_{\alpha}\right) \longrightarrow(M, L)
$$

that is required to be either a holomorphic disc $u_{\alpha}:(\mathbb{D}, \partial \mathbb{D}) \rightarrow(M, L)$, a holomorphic sphere $u_{\alpha}: \mathbb{C} P^{1} \rightarrow M$, or a holomorphic sphere $u_{\alpha}:\left(\mathbb{C} P^{1}, \infty\right) \rightarrow(M, L)$ that maps the point at infinity to $L$.

To each edge $\{\alpha, \beta\}$ we associate two points $z_{\alpha \beta} \in \Sigma_{\alpha}$ and $z_{\beta \alpha} \in \Sigma_{\beta}$ which we call nodal points. If $z_{\alpha \beta}$ is a boundary point on $\Sigma_{\alpha}=\mathbb{D}$ then $\Sigma_{\beta}=\mathbb{D}$ is a disc and $z_{\beta \alpha}$ is a boundary point too. In other words, the nodal points corresponding to an edge are either interior or boundary points; no interior point can correspond to a boundary point and vice versa. We require that for different edges $\{\alpha, \beta\}$ and $\{\alpha, \gamma\}$ the points $z_{\alpha \beta}$ and $z_{\alpha \gamma}$ on $\Sigma_{\alpha}$ are different. If the surface $\left(\Sigma_{\alpha}, \Gamma_{\alpha}\right)$ is the pointed $\left(\mathbb{C} P^{1}, \infty\right)$ we require all nodal points on $\Sigma_{\alpha}$ to be different from $\infty$.

The boundary tree $\partial T$ of $T$ is the subtree, whose vertices $\alpha$ correspond to surfaces $\Sigma_{\alpha}$ with $\Gamma_{\alpha} \neq \emptyset$. We require that $\partial T$ is non-empty. The boundary tree consists of a single vertex if $\Gamma_{\alpha}=\infty$ for some $\alpha \in V$.

The collection of holomorphic maps $u_{\alpha}$ is required to satisfy $u_{\alpha}\left(z_{\alpha \beta}\right)=u_{\beta}\left(z_{\beta \alpha}\right)$ for all nodal points. Passing to the quotient of the disjoint union of the $\Sigma_{\alpha}$ by the set of all nodal points we obtain a nodal surface of genus zero with connected boundary that eventually is collapsed to one point. The $u_{\alpha}$ pass to a holomorphic map $\mathbf{u}$ on the quotient.

We denote the set of nodal points on $\Sigma_{\alpha}$ by $Z_{\alpha}$ including $\infty$ in the pointed case. The map $\mathbf{u}$ is called stable if for all vertices $\alpha$ for which the map $u_{\alpha}$ is constant the number of nodal points is $\# Z_{\alpha} \geq 3$ (regardless $\Sigma_{\alpha}$ is a disc or a sphere) or $Z_{\alpha}$ consists of two points that do not both lie on the boundary $\Gamma_{\alpha}=\partial \mathbb{D}$ in the case that $\Sigma_{\alpha}=\mathbb{D}$ is a disc.

1.4. Energy of a bubble tree. Let $\mathbf{u}=\left(u_{\alpha}\right)$ be a stable map with tree $T=$ $(V, E)$. Removing (the interior of) an edge $\{\alpha, \beta\} \in E$ decomposes $T$ into two subtrees. Denote by $T_{\alpha \beta}$ the component that has $\beta$ as a vertex. Observe that any subtree of $T$ is of the form $T_{\alpha \beta}$. The energy of the bubble tree $T_{\alpha \beta}$ is

$$
E_{\alpha \beta}(\mathbf{u})=\sum_{\gamma \text { is vertex of } T_{\alpha \beta}} E\left(u_{\gamma}\right) .
$$

Similarly, the total energy is

$$
E(\mathbf{u})=\sum_{\alpha \in V} E\left(u_{\alpha}\right) .
$$


1.5. Gromov convergence. We say that a sequence of holomorphic discs Gromov converges to a stable map $\mathbf{u}$ (denoting the underlying tree by $T=(V, E)$ ) provided that for each $\alpha \in V$ there exists a sequence of Möbius transformations $\varphi_{\alpha}^{\nu}$ in the automorphism group of $\Sigma_{\alpha}$ such that the following holds: If $\Sigma_{\alpha}$ is the disc $\mathbb{D}$ or the unpointed $\mathbb{C} P^{1}$ we require:

- (Rescaling): For all $\{\alpha, \beta\} \in E$ the sequence $\left(\varphi_{\alpha}^{\nu}\right)^{-1} \circ \varphi_{\beta}^{\nu}$ converges to the constant map $z_{\alpha \beta}$ in $C_{\mathrm{loc}}^{\infty}$ on $\Sigma_{\beta} \backslash\left\{z_{\beta \alpha}\right\}$.

- (Map): For all $\alpha \in V$ the sequence

$$
u_{\alpha}^{\nu}:=u^{\nu} \circ \varphi_{\alpha}^{\nu}
$$

converges to $u_{\alpha}$ in $C_{\mathrm{loc}}^{\infty}$ on $\Sigma_{\alpha} \backslash Z_{\alpha}$.

- (Energy): For all $\{\alpha, \beta\} \in E$

$$
E_{\alpha \beta}(\mathbf{u})=\lim _{\varepsilon \rightarrow \infty} \lim _{\nu \rightarrow \infty} E\left(u_{\alpha}^{\nu} ; B_{\varepsilon}\left(z_{\alpha \beta}\right)\right),
$$

where $B_{\varepsilon}\left(z_{\alpha \beta}\right)$ denotes set of points in $\Sigma_{\alpha}$ with distance less than $\varepsilon$ from $z_{\alpha \beta}$ and

$$
E\left(u_{\alpha}^{\nu} ; B_{\varepsilon}\left(z_{\alpha \beta}\right)\right)=\int_{B_{\varepsilon}\left(z_{\alpha \beta}\right)}\left(u_{\alpha}^{\nu}\right)^{*} \omega .
$$

If the degenerate case $\left(\Sigma_{\alpha}=\mathbb{C} \cup \infty, \Gamma_{\alpha}=\infty\right)$ appears we require in addition that:

- (Rescaling): There exists for each compact subset $K \subset \mathbb{C}$ a natural number $\nu_{0}$ such that $\varphi_{\alpha}^{\nu}(K) \subset B_{1}(0)$ for all $\nu \geq \nu_{0}$.

- (Energy): For $\infty \in \Sigma_{\alpha}$

$$
\lim _{\varepsilon \rightarrow \infty} \lim _{\nu \rightarrow \infty} E\left(u_{\alpha}^{\nu} ; B_{\varepsilon}(\infty) \cap\left(\varphi_{\alpha}^{\nu}\right)^{-1}(\mathbb{D})\right)=0,
$$

where $B_{\varepsilon}(\infty)$ denotes set of points in $\Sigma_{\alpha}$ with distance less than $\varepsilon$ from $\infty$.

\section{LAGRANGEFY}

The bubbling off phenomenon of holomorphic curves is similar to the one of minimal surfaces. A mean value inequality holds true provided the energy is sufficiently small. In the presence of totally real boundary conditions the mean value inequality can be obtained via Schwarz reflection of the energy density with respect to a suitable local metric, see [6, p. 265] and cf. Section 2.2. In order to obtain the removable singularity theorem it is necessary that certain boundary terms during partial integrations via Stokes's theorem vanish. One way to achieve this is a choice of a local symplectic form that turns the boundary condition into a Lagrangian submanifold as observed in 28, see Section 2.1. After all the convergence modulo bubbling theorem from [22] passes to the present situation, see Section 2.5.

Denote by $K_{M} \subset M$ and $K_{L} \subset L$ relative compact open subsets such that $u^{\nu}(\mathbb{D}) \subset K_{M}$ and $u^{\nu}(\partial \mathbb{D}) \subset K_{L}$ for all $\nu \in \mathbb{N}$.

2.1. Local symplectic form. By [9, p. 544/545] there exists a relative compact open domain $\Omega_{L}$ in $M$ and a symplectic form $\omega_{L}$ on a neighbourhood of the closure of $\Omega_{L}$ such that $\Omega_{L}$ contains $K_{L}$ and $\omega_{L}$

- tames the restriction of $J$ to $\Omega_{L}$,

- has a primitive $\lambda$ that vanishes on $L$, and

- turns $J T\left(L \cap \Omega_{L}\right)$ into a Lagrangian subbundle of $T \Omega_{L}$. 
Notice, that the metrics obtained by symmetrising $\omega(., J$.$) and \omega_{L}(., J$.$) re-$ stricted to $\Omega_{L}$ are equivalent.

The local symplectic form is constructed in the following way: Let $g$ be a metric on $M$ such that $J$ is a orthogonal transformation and the subbundle $T L$ of $\left.T M\right|_{L}$ is orthogonal to $J T L$ along a neighbourhood of $K_{L}$. Define a non-degenerate 2 -form $\sigma:=g(J .,$.$) and notice that \sigma$ tames $J$. The normal exponential map of $g$ allows an identification of a neighbourhood $\Omega_{L}$ of $K_{L}$ with a neighbourhood of $K_{L}$ in $T^{*} L$, cf. the proof of [21, Theorem 3.33]. Under this identification the restriction of $\sigma$ to $L \cap \Omega_{L}$ equals $\mathrm{d} \lambda$, where $\lambda$ denotes the Liouville form given by $T^{*} L$. The claim follows by setting $\omega_{L}=\mathrm{d} \lambda$ and shrinking $\Omega_{L}$ if necessary.

2.2. Mean value inequality. We denote by $B_{r}(z)$ the set of complex numbers in the closed upper half-plane $\mathbb{H}$ whose Euclidean distance to $z \in \mathbb{H}$ is smaller than $r$. Then there exist positive constants $\hbar$ and $C$ such that for all holomorphic maps

$$
w:(\mathbb{H}, \mathbb{R}) \longrightarrow\left(K_{M}, K_{L}\right)
$$

with

$$
\frac{1}{2} \int_{B_{r}(z)}|\nabla w|^{2}<\hbar
$$

the mean value inequality

$$
|\nabla w(z)|^{2} \leq \frac{C}{r^{2}} \int_{B_{r}(z)}|\nabla w|^{2}
$$

is satisfied. This follows from [6, Appendix A] or [22, Section 4.3] in the totally real case also even if the results are only stated for Lagrangian boundaries.

In particular, all non-constant holomorphic spheres $\mathbb{C} P^{1} \rightarrow K_{M}$ and all nonconstant holomorphic discs $(\mathbb{D}, \partial \mathbb{D}) \rightarrow\left(K_{M}, K_{L}\right)$ have energy greater or equal to $\hbar$. Indeed, because of the conformal invariance of the energy we can assume that $w$ is defined on $\mathbb{H}$ or $\mathbb{C}$, resp. Consider $B_{r}(z)$ for each point $z$ in $B_{r}(0)$. If the energy of $w$ on $B_{2 r}(0)$ is smaller than $\hbar$ then $\sup _{B_{r}(0)}|\nabla w|^{2}$ is bounded by the energy of $w$ on $B_{2 r}(0)$ times $2 C / r^{2}$. The claim follows if this inference is conclusive for arbitrary large radii $r$.

2.3. Convention. The mean value inequality estimates the gradient of a holomorphic map in terms of its $L^{2}$-norm provided the energy is less than $\hbar$. Because our applications will be geometric in nature the holomorphic maps to which we will apply the inequality will have even smaller energy. Therefore, we shrink $\hbar$ such that:

- The quantity $\sqrt{8 C \hbar} \pi$ is smaller than the distance of $K_{L}$ and the boundary of $\Omega_{L}$.

- There exists a positive constant $c$ such that for any smooth curve $\gamma$ with endpoints $\gamma(0)$ and $\gamma(1)$ on $K_{L}$ and length $(\gamma)$ less than or equal to $\sqrt{8 C \hbar} \pi$ the isoperimetric inequality holds true in the sense that

$$
\left|\int_{0}^{1} \gamma^{*} \lambda\right| \leq c \operatorname{length}(\gamma)^{2},
$$

as well as the corresponding isoperimetric inequality for smooth loops, see [22, Theorem 4.4.1]. Notice, that the inequality is invariant under reparametrisations. 
2.4. Removal of singularity. Let $w$ be a punctured holomorphic half-plane

$$
w:(\mathbb{H} \backslash 0, \mathbb{R} \backslash 0) \longrightarrow\left(K_{M}, K_{L}\right)
$$

or a punctured holomorphic plane $w: \mathbb{C} \backslash 0 \rightarrow K_{M}$ of finite energy. Then $w$ extends to a holomorphic map on $\mathbb{H}$, resp., $\mathbb{C}$.

To see this notice that

$$
E\left(w ; B_{r}(0)\right)=\int_{B_{r}(0)} w^{*} \omega
$$

tends to zero for $r \searrow 0$. Using polar coordinates we consider $\operatorname{arcs} \gamma_{r}(\theta)=w\left(r \mathrm{e}^{\mathrm{i} \theta}\right)$, for $\theta \in[0, \pi]$, that lie on the holomorphic half-plane $w$. Because of

$$
\left|\dot{\gamma}_{r}(\theta)\right|^{2}=\frac{r^{2}}{2}\left|\nabla w\left(r \mathrm{e}^{\mathrm{i} \theta}\right)\right|^{2}
$$

and $B_{r}\left(r \mathrm{e}^{\mathrm{i} \theta}\right) \subset B_{2 r}(0)$ the mean value inequality implies

$$
\left|\dot{\gamma}_{r}(\theta)\right|^{2} \leq \frac{C}{2} \int_{B_{2 r}(0)}|\nabla w|^{2}
$$

provided that $r$ is sufficiently small. Therefore, the length of $\gamma_{r}$ tends to zero so that $w$ maps a neighbourhood of $0 \in \mathbb{H}$ into $\Omega_{L}$.

Observe that up to this point the exact local symplectic form $\omega_{L}$ is not used. The removability of the singularity follows with [22, Theorem 4.1.2], which requires a Lagrangian boundary condition in the case of the half-plane. Using the symplectic form $\omega_{L}$ on $\Omega_{L}$ this requirement will be satisfied.

2.5. Convergence modulo bubbling. Let $\Omega^{\nu}$ be exhausting sequence of open subsets of $\mathbb{H}$. Let $w^{\nu}:\left(\Omega^{\nu}, \Omega^{\nu} \cap \mathbb{R}\right) \rightarrow\left(K_{M}, K_{L}\right)$ be a sequence of holomorphic maps with bounded energy. With the results of the proceeding section one shows as in [22, Section 4.6]: There exists a holomorphic map $w:(\mathbb{H}, \mathbb{R}) \rightarrow\left(K_{M}, K_{L}\right)$ and a finite set $Z=\left\{z_{1}, \ldots, z_{\ell}\right\}$ of points in $\mathbb{H}$, the so-called bubble points, such that

- $w^{\nu}$ converges to $w$ in $C_{\mathrm{loc}}^{\infty}$ on $\mathbb{H} \backslash Z$.

- For all bubble points $z_{j}$ and for all $\varepsilon>0$ such that $B_{\varepsilon}\left(z_{j}\right) \cap Z$ consists of precisely the bubble point $z_{j}$ the limit

$$
m_{\varepsilon}\left(z_{j}\right):=\lim _{\nu \rightarrow \infty} E\left(w^{\nu} ; B_{\varepsilon}\left(z_{j}\right)\right)
$$

exists. Moreover the map $\varepsilon \mapsto m_{\varepsilon}\left(z_{j}\right)$ is continuous and the mass $m_{j}$ of the bubble point $z_{j}$ satisfies

$$
m_{j}:=\lim _{\varepsilon \rightarrow 0} m_{\varepsilon}\left(z_{j}\right) \geq \hbar .
$$

- For all relative compact subsets $K$ of $\mathbb{H}$ that contain the bubble set $Z$

$$
\lim _{\nu \rightarrow \infty} E\left(w^{\nu} ; K\right)=E(w ; K)+m_{1}+\ldots+m_{\ell} .
$$

The analog statement with $\mathbb{H}$ replaced by $\mathbb{C}$ holds true. 


\section{Bubbles Connect}

In this section we will prove Theorem 1.1. The argumentation is the same as in [6. Section 3.2.2] and the reader is referred to the cited source. Only modifications of the bubbling off analysis caused by the totally real boundary condition are required. The implementation of the modifications is the aim of this section.

A sequence of holomorphic discs as described in the theorem converges modulo bubbling to a holomorphic disc, see Section 2.5. A suitable rescaling in a neighbourhood of any of the bubble points yields sequences of holomorphic maps that again converge modulo bubbling. The proof proceeds by induction, which terminates after finitely many steps due to the energy bound. Care has to be taken in view of the stability condition, see [6. Section 3.2.2].

The rescaling near the bubble points is done in a particular way, see Section 3.2. In order to obtain a stable map for the limiting object it is necessary to detect all the bubbles. The main tool for that is the concentration inequality of Section 3.1. It implies that the energy of the cut off annuli that have large ratio is concentrated near the boundary components. In order to show that the bubbles connect the distance-energy inequality is provided in Section 3.1 also. That the relative homotopy class is preserved under Gromov convergence will follow with the distance-energy inequality too, see [6, Section 3.3].

3.1. Long cylinders with small energy. We consider a sequence of holomorphic maps $w^{\nu}:\left(B_{1}(0),(-1,1)\right) \rightarrow(M, L)$ that are defined on the open unit half-disc in $\mathbb{H}$. We assume that $w^{\nu}\left(B_{1}(0)\right) \subset K_{M}$ and $w^{\nu}((-1,1)) \subset K_{L}$ for all $\nu \in \mathbb{N}$. Moreover, we consider sequences $\varepsilon^{\nu}, \delta^{\nu}$ of positive real numbes and a sequence of complex numbers $z^{\nu}=x^{\nu}+\mathrm{i} y^{\nu}$ on the upper half-plane such that

- $\delta^{\nu} \leq \varepsilon^{\nu} \searrow 0$,

- $\varepsilon^{\nu} / \delta^{\nu} \rightarrow \infty$,

- $z^{\nu}$ converges to 0 in $\mathbb{H}$, and

- there exists a positive constant $\eta$ such that $0 \leq y^{\nu} / \delta^{\nu} \leq \eta$.

We assume that the energy

$$
e\left(w^{\nu}\right):=E\left(w^{\nu} ; A_{z^{\nu}}\left(\delta^{\nu}, \varepsilon^{\nu}\right)\right)<\hbar
$$

for all $\nu \in \mathbb{N}$, where

$$
A_{z}(\delta, \varepsilon):=B_{\varepsilon}(z) \backslash \overline{B_{\delta}(z)} \subset \mathbb{H}
$$

is an open annulus cut off along the real line.

We remark that for all $T>\ln \eta$ we have $y^{\nu}<\mathrm{e}^{T} \delta^{\nu}$ so that both circular boundary components of $A_{z^{\nu}}\left(\mathrm{e}^{T} \delta^{\nu}, \mathrm{e}^{-T} \varepsilon^{\nu}\right)$ intersect the real axis. Moreover, the annulus $A_{z^{\nu}}\left(\mathrm{e}^{T} \delta^{\nu}, \mathrm{e}^{-T} \varepsilon^{\nu}\right)$ is non-empty precisely if $T<\ln \sqrt{\varepsilon^{\nu} / \delta^{\nu}}$.

Lemma 3.1. There exist positive constants $c$ (only depending on the geometry of $K_{L}$ and $K_{M}$ ), $T_{0}$ (only depending on $\eta$ ), and a natural number $\nu_{0}$ such that for all $\nu \geq \nu_{0}$ (we have $\ln \sqrt{\varepsilon^{\nu} / \delta^{\nu}}>T_{0}$ ) and for all $T \in\left[T_{0}, \ln \sqrt{\varepsilon^{\nu} / \delta^{\nu}}\right]$ the following inequalities hold true, where we abbreviate $w=w^{\nu}, z=z^{\nu} \varepsilon=\varepsilon^{\nu}$, and $\delta=\delta^{\nu}$ :

\section{- (Concentration inequality):}

$$
E\left(w ; A_{z}\left(\mathrm{e}^{T} \delta, \mathrm{e}^{-T} \varepsilon\right)\right) \leq c \mathrm{e}^{-T / c} e(w)
$$


- (Distance-energy inequality): For all points $z_{1}, z_{2}$ on the cut off annulus $A_{z}\left(\mathrm{e}^{T} \delta, \mathrm{e}^{-T} \varepsilon\right)$ we have that

$$
\operatorname{dist}\left(w\left(z_{1}\right), w\left(z_{2}\right)\right) \leq c \mathrm{e}^{-T / c} \sqrt{e(w)} .
$$

Remark 3.2. As the proof will show we can take $T_{0}=\max \{7,3+2 \ln \eta\}$.

Proof. In order to symmetrise the problem we rescale

$$
v(\zeta):=w(\sqrt{\delta \varepsilon} \cdot \zeta)
$$

so that the resulting holomorphic map $v$ is defined on $B_{1 / \sqrt{\delta \varepsilon}}(0)$. Notice, that the inverse rescaling map sends $A_{z}(\delta, \varepsilon)$ to

$$
A(1 / R, R):=A_{z / \sqrt{\delta \varepsilon}}(1 / R, R),
$$

where $R:=\sqrt{\varepsilon / \delta}$. By conformal invariance and invariance of the length under reparametrisations it suffices to prove the symmetrised variant of the lemma. In order to simplify the notation we will write $z$ instead of $z / \sqrt{\delta \varepsilon}$.

The choice $T_{0}>\ln \eta$ is understood so that for all $T \geq T_{0}$ both circular boundary components of the cut off annuli $A\left(\mathrm{e}^{T} / R, R / \mathrm{e}^{T}\right)$ intersect the real axis. We claim that for $T$ sufficiently large $v$ maps the annuli $A\left(\mathrm{e}^{T} / R, R / \mathrm{e}^{T}\right)$ into $\Omega_{L}$. For that we consider the curves

$$
\gamma_{r}(\theta):=v\left(z+r \mathrm{e}^{\mathrm{i} \theta}\right) .
$$

In order to estimate its length we cover the $\operatorname{arcs} \theta \mapsto z+r \mathrm{e}^{\mathrm{i} \theta}$ by the $r / 2$-distance $\operatorname{discs} B_{r / 2}\left(z+r \mathrm{e}^{\mathrm{i} \theta}\right)$ of $\mathbb{H}$. We require that $r \in(2 / R, 2 R / 3)$ so that the cut off discs $B_{r / 2}\left(z+r \mathrm{e}^{\mathrm{i} \theta}\right)$ are contained in $A(1 / R, R)$. Hence, the energy of $v$ restricted to the $r / 2$-distance discs is bounded by $\hbar$. The mean value inequality implies

$$
\left|\dot{\gamma}_{r}(\theta)\right|^{2} \leq 2 C \int_{A(1 / R, R)}|\nabla v|^{2} .
$$

Therefore, the length of $\gamma_{r}$ is bounded by $2 \pi \sqrt{2 C \hbar}$. We choose $T_{0}>\ln 2$ so that $A\left(\mathrm{e}^{T} / R, R / \mathrm{e}^{T}\right)$ is contained in $A(2 / R, 2 R / 3)$ for all $T \geq T_{0}$. This implies that

$$
\text { length }\left(\gamma_{r}\right) \leq \sqrt{8 C \hbar} \pi
$$

for all $r \in\left(\mathrm{e}^{T} / R, R / \mathrm{e}^{T}\right)$. The curves $\gamma_{r}$ connect interior points of the holomorphic square

$$
v\left(A\left(\mathrm{e}^{T} / R, R / \mathrm{e}^{T}\right)\right)
$$

to the boundary in $K_{L}$ provided $T \geq T_{0}$. By the assumptions on the quantity $\hbar$, see Section 2.3, the holomorphic square is contained in $\Omega_{L}$ as claimed.

The closure of $\Omega_{L}$ is compact. Hence, up to a change of constants in the formulation of the lemma it suffices to prove the inequalities with respect to the symplectic form $\omega_{L}=\mathrm{d} \lambda$ and the metric given by the symmetrisation of $\omega_{L}(., J$. ).

In view of the first inequality we will use logarithmic polar coordinates. We reparametrise

$$
u(s+\mathrm{i} t)=v\left(z+\mathrm{e}^{s+\mathrm{i} t}\right) .
$$

The preimage $D_{T}$ of $A\left(\mathrm{e}^{T} / R, R / \mathrm{e}^{T}\right)$ in the square

$$
(T-\ln R, \ln R-T) \times\left(-\frac{\pi}{2}, \frac{3}{2} \pi\right)
$$


is bounded by the graphs of the functions $s \mapsto t_{0}(s), t_{1}(s)$, which are mapped into $K_{L}$ by $u$. Therefore, we can express the energy

$$
e(T)=\int_{D_{T}} u^{*} \mathrm{~d} \lambda
$$

in terms of $\gamma_{s}(t)=u(s+\mathrm{i} t)$ as

$$
e(T)=\int\left(\gamma_{\ln R-T}\right)^{*} \lambda-\int\left(\gamma_{T-\ln R}\right)^{*} \lambda,
$$

where the first integral is taken over all $t$ in $\left(t_{0}(\ln R-T), t_{1}(\ln R-T)\right)$; the second over all $t$ in $\left(t_{0}(T-\ln R), t_{1}(T-\ln R)\right)$. With the isoperimetric inequality, see Section 2.3, this yields

$$
e(T) \leq c_{1}\left(\text { length }\left(\gamma_{\ln R-T}\right)^{2}+\operatorname{length}\left(\gamma_{T-\ln R}\right)^{2}\right)
$$

for a universal constant $c_{1}>0$. Using the Cauchy-Schwarz inequality and the Cauchy-Riemann equation we obtain

$$
e(T) \leq 2 \pi c_{1}\left(\int\left|u_{s}(\ln R-T+\mathrm{i} t)\right|^{2} \mathrm{~d} t+\int\left|u_{s}(T-\ln R+\mathrm{i} t)\right|^{2} \mathrm{~d} t\right),
$$

where the first integral is taken over $\left(t_{0}(\ln R-T), t_{1}(\ln R-T)\right)$; the second over $\left(t_{0}(T-\ln R), t_{1}(T-\ln R)\right)$. Taking the derivative of

$$
e(T)=\int_{T-\ln R}^{\ln R-T}\left(\int_{t_{0}(s)}^{t_{1}(s)}\left|u_{s}(s+\mathrm{i} t)\right|^{2} \mathrm{~d} t\right) \mathrm{d} s
$$

with respect to $T$ yields

$$
\frac{\mathrm{d}}{\mathrm{d} T} e(T) \leq-\frac{1}{2 \pi c_{1}} e(T)
$$

for all $T \geq T_{0}$. Grönwall's inequality implies

$$
e(T) \leq \mathrm{e}^{-\frac{1}{2 \pi c_{1}}\left(T-T_{0}\right)} e\left(T_{0}\right) .
$$

Because $e\left(T_{0}\right)$ is bounded by $e(w)$ up to a universal constant this proves the concentration inequality; the first inequality of the lemma.

We prove the second. Observe that each two points

$$
z_{1}=z+r_{1} \mathrm{e}^{\mathrm{i} \theta_{1}}, \quad z_{2}=z+r_{2} \mathrm{e}^{\mathrm{i} \theta_{2}}
$$

in $A\left(\mathrm{e}^{T} / R, R / \mathrm{e}^{T}\right)$ can be joint by a circular arc provided $r_{1}=r_{2}$, or, if $r_{1} \neq r_{2}$, by a path consisting of a circular arc connecting $z_{1}$ with $z+r_{1}$ i, a line segment connecting $z+r_{1} \mathrm{i}$ with $z+r_{2} \mathrm{i}$, and a circular arc connecting $z+r_{2} \mathrm{i}$ with $z_{2}$. Therefore, it is possible to prove the second inequality with help of circular arcs and line segments in $z+\mathbb{R i}$ only.

We estimate the length of the path

$$
I \ni \theta \longmapsto v\left(z+r \mathrm{e}^{\mathrm{i} \theta}\right),
$$

where $I$ is a subinterval of $\left(-\frac{\pi}{2}, \frac{3}{2} \pi\right)$ and $r \in\left(\mathrm{e}^{T} / R, R / \mathrm{e}^{T}\right)$. By the mean value inequality

$$
\left|\frac{\partial v}{\partial \theta}\left(z+r \mathrm{e}^{\mathrm{i} \theta}\right)\right|=\frac{r}{\sqrt{2}}\left|\nabla v\left(z+r \mathrm{e}^{\mathrm{i} \theta}\right)\right|
$$


is bounded by

$$
\sqrt{2 C}\left(\int_{B_{r / 2}\left(z+r \mathrm{e}^{\mathrm{i} \theta}\right)}|\nabla v|^{2}\right)^{1 / 2} .
$$

For all $T \geq \ln 2+T_{0}$ we get with $S:=T-\ln 2$ that $B_{r / 2}\left(z+r \mathrm{e}^{\mathrm{i} \theta}\right)$ is contained in

$$
A\left(\frac{1}{2} \frac{\mathrm{e}^{T}}{R}, \frac{3}{2} \frac{R}{\mathrm{e}^{T}}\right)=A\left(\frac{\mathrm{e}^{S}}{R}, \frac{3}{4} \frac{R}{\mathrm{e}^{S}}\right)
$$

the latter being contained in $A\left(\mathrm{e}^{T_{0}} / R, R / \mathrm{e}^{T_{0}}\right)$. Hence, we can estimate the upper bound by

$$
2 \sqrt{C} \sqrt{E\left(v ; A\left(\mathrm{e}^{S} / R, R / \mathrm{e}^{S}\right)\right)}
$$

which by the concentration inequality is bounded by

$$
2 \sqrt{C} \sqrt{c \mathrm{e}^{-S / c} e(w)}
$$

Consequently, replacing $T_{0}$ by $T_{0}+\ln 2$ we find a constant $c_{2}>0$ such that

$$
\left|\frac{\partial v}{\partial \theta}\left(z+r \mathrm{e}^{\mathrm{i} \theta}\right)\right| \leq c_{2} \mathrm{e}^{-T / c_{2}} \sqrt{e(w)}
$$

for all $T \geq T_{0}$. Integrating $\theta$ over the subinterval $I$ proves the distance-energy inequality for circular paths.

We prove the distance-energy inequality along line segments $r \mapsto z+r$ i with $r \in\left(\mathrm{e}^{T} / R, R / \mathrm{e}^{T}\right)$. Using logarithmic polar coordinates we write

$$
u(s+\mathrm{i} t)=v\left(z+\mathrm{e}^{s+\mathrm{i} t}\right),
$$

where $|s|<\ln R-T$ and $t \in(0, \pi)$. We choose $T_{0}>\pi / 2$. The mean value inequality implies that

is bounded by

$$
\left|\frac{\partial u}{\partial s}(s+\mathrm{i} \pi / 2)\right|=\frac{1}{\sqrt{2}}|\nabla u(s+\mathrm{i} \pi / 2)|
$$

$$
\frac{\sqrt{2 C}}{\pi}\left(\int_{B_{\pi / 2}(s+\mathrm{i} \pi / 2)}|\nabla u|^{2}\right)^{1 / 2} .
$$

Notice, that $B_{\pi / 2}(s+\mathrm{i} \pi / 2)$ is contained in the square

$$
(-|s|-\pi / 2,|s|+\pi / 2) \times(0, \pi) .
$$

The image under the inverse conformal coordinate transformation is contained in

$$
A:=A\left(\mathrm{e}^{-|s|-\pi / 2}, \mathrm{e}^{|s|+\pi / 2}\right)=A\left(\mathrm{e}^{\ln R-|s|-\pi / 2} / R, R / \mathrm{e}^{\ln R-|s|-\pi / 2}\right) .
$$

Therefore,

$$
\left|\frac{\partial u}{\partial s}(s+\mathrm{i} \pi / 2)\right| \leq \frac{2}{\pi} \sqrt{C} \sqrt{E(v ; A)} .
$$

Further, we are allowed to apply the concentration inequality for all $T \geq T_{0}+\pi / 2$. Indeed, we get $|s|<\ln R-T_{0}-\pi / 2$ such that

$$
A \subset A\left(\mathrm{e}^{T_{0}} / R, R / \mathrm{e}^{T_{0}}\right) .
$$


Hence, we obtain

$$
\left|\frac{\partial u}{\partial s}(s+\mathrm{i} \pi / 2)\right| \leq \frac{2}{\pi} \sqrt{C} \sqrt{c \mathrm{e}^{(|s|+\pi / 2-\ln R) / c} e(w)} .
$$

An integration of the inequality over all $s \in(T-\ln R, \ln R-T)$ using the symmetry of the integrand on the right hand side shows that the length of the path $s \mapsto$ $u(s+\mathrm{i} \pi / 2)$ is bounded by

$$
\frac{2}{\pi} \sqrt{C} \sqrt{c e(w)} 4 c \mathrm{e}^{(\pi / 2-T) / 2 c} .
$$

Replacing $T_{0}$ by $T_{0}+\pi / 2$ completes the proof of the lemma.

3.2. Soft rescaling. Consider the open disc $B_{r}\left(z_{0}\right)$ of radius $r>0$ in $\mathbb{H}$ with center equal to $z_{0} \in \mathbb{H}$ and a sequence $w^{\nu}:\left(B_{r}\left(z_{0}\right), B_{r}\left(z_{0}\right) \cap \mathbb{R}\right) \rightarrow\left(K_{M}, K_{L}\right)$ of holomorphic maps with bounded energy. Assume that there exists a pointed limiting holomorphic map $w:\left(B_{r}\left(z_{0}\right), B_{r}\left(z_{0}\right) \cap \mathbb{R}\right) \rightarrow\left(K_{M}, K_{L}\right)$ in the sense that

- $w^{\nu}$ converges to $w$ in $C_{\mathrm{loc}}^{\infty}$ on $B_{r}\left(z_{0}\right) \backslash\left\{z_{0}\right\}$ and

- the limit

$$
m_{0}=\lim _{\varepsilon \rightarrow 0} \lim _{\nu \rightarrow \infty} E\left(w^{\nu} ; B_{\varepsilon}\left(z_{0}\right)\right)
$$

the so-called mass at $z_{0}$, exists and is greater or equal to $\hbar$.

The aim of this section is to describe the behavior of the sequence $w^{\nu}$ near the selected bubble point $z_{0}$. We will use the so-called soft rescaling technique that was introduced in [16]. Denote by $\Sigma$ the closed unit disc $\mathbb{D}=\mathbb{H} \cup \infty$ or $\mathbb{C} P^{1}=\mathbb{C} \cup \infty$ depending on whether $z_{0}$ is real or has positive imaginary part.

Lemma 3.3. There exists a sequence $\varphi^{\nu}$ of Möbius transformations of $\Sigma$, a holomorphic map $v:(\mathbb{D}, \partial \mathbb{D}) \rightarrow\left(K_{M}, K_{L}\right)$, resp., $v: \mathbb{C} P^{1} \rightarrow K_{M}$, and a finite subset $Z=\left\{z_{1}, \ldots, z_{\ell}\right\}$ of bubble points on $\Sigma \backslash \infty$ such that

- A subsequence of $v^{\nu}:=w^{\nu} \circ \varphi^{\nu}$ converges to $v$ in $C_{\mathrm{loc}}^{\infty}$ on $\Sigma \backslash(Z \cup \infty)$ and $\varphi^{\nu}$ converges to 0 in $C_{\mathrm{loc}}^{\infty}$ on $\Sigma \backslash \infty$.

- If $v$ is constant, then the following stability condition is satisfied: Either $\# Z \geq 2$, regardless whether $\Sigma$ is a disc or a sphere, or $\# Z<2$, in which case $\Sigma$ is the closed unit disc and there is precisely one bubble point in $Z$, which lies in the interior.

- For all bubble points $z_{j}$ the limit

$$
m_{j}:=\lim _{\varepsilon \rightarrow 0} \lim _{\nu \rightarrow \infty} E\left(v^{\nu} ; B_{\varepsilon}\left(z_{j}\right)\right)
$$

the so-called mass at $z_{j}$, exists and is greater or equal to $\hbar$.

- Denote by

$$
m_{\infty}:=\lim _{R \rightarrow \infty} \lim _{\nu \rightarrow \infty} E\left(v^{\nu} ; \Sigma \backslash B_{R}(0)\right)
$$

the mass at infinity. Then

$$
\lim _{\nu \rightarrow \infty} E\left(w^{\nu}\right)=m_{0}+m_{\infty}
$$

and

$$
m_{0}=E(v)+m_{1}+\ldots+m_{\ell}
$$

so that no energy is lost.

- The bubbles connect, i.e., $w(0)=v(\infty)$. 
Proof. In the case the distinguished bubble point $z_{0}$ does not lie on the real axis the proof can be found in [22, Section 4.7]. In the alternative case we will translate the arguments from [6. Section 3.2.1] to the present situation. The modifications are caused by the totally real boundary condition.

We can assume that $z_{0}=0$ and $r=2$. The sequence

$$
z^{\nu}=x^{\nu}+\mathrm{i} y^{\nu}
$$

of points on which $\left|\nabla w^{\nu}\right|$ attains its maximum on $B_{1}(0)$ converges to the origin. For $\nu$ sufficiently large we find $\delta^{\nu}>0$ such that

$$
E\left(w^{\nu} ; B_{\delta^{\nu}}\left(z^{\nu}\right)\right)=m_{0}-\hbar / 2 .
$$

By the definition of $m_{0}$ the sequence $\delta^{\nu}$ must converge to 0 . We will consider two cases. Either a subsequence of $y^{\nu} / \delta^{\nu}$ converges to a real number or tends to infinity.

We start with the first case. We can assume that $y^{\nu} / \delta^{\nu}$ converges to $\eta \geq 0$. Setting

$$
\varphi^{\nu}(z):=x^{\nu}+\delta^{\nu} z
$$

for all $\nu$ we obtain a sequence of Möbius transformations, which preserve the upper half-plane. The sequence $\varphi^{\nu}$ tends to 0 in $C_{\mathrm{loc}}^{\infty}$. With Section 2.5 the sequence

$$
v^{\nu}:=w^{\nu} \circ \varphi^{\nu}
$$

converges modulo bubbling in the complement $\mathbb{H} \backslash Z$ of finitely many bubble points $Z=\left\{z_{1}, \ldots, z_{\ell}\right\}$ to $v$. The masses exists at all bubble points $z_{j}$. By Section $2.4 v$ extends to a holomorphic disc.

By construction

$$
E\left(v^{\nu} ; B_{1}\left(\mathrm{i} y^{\nu} / \delta^{\nu}\right)\right)=m_{0}-\hbar / 2
$$

The definition of $m_{0}$ implies that for $\varepsilon>0$ sufficiently small and for $\nu \in \mathbb{N}$ sufficiently large

$$
E\left(v^{\nu} ; B_{\varepsilon / \delta^{\nu}}\left(-x^{\nu} / \delta^{\nu}\right)\right) \leq m_{0}+\hbar / 4
$$

Because the masses are greater or equal than $\hbar$ the bubble points $z_{1}, \ldots, z_{\ell}$ are contained in the closure of $B_{1}(\mathrm{i} \eta)$. Moreover, because the supremum of $\left|\nabla v^{\nu}\right|$ over $B_{1 / \delta^{\nu}}\left(-x^{\nu} / \delta^{\nu}\right)$ is attained at $\mathrm{i} y^{\nu} / \delta^{\nu}$ the point i $\eta$ is a bubble point provided $Z$ is not empty.

We claim that

$$
\lim _{R \rightarrow \infty} \lim _{\nu \rightarrow \infty} E\left(v^{\nu} ; B_{R}\left(\mathrm{i} y^{\nu} / \delta^{\nu}\right)\right)=m_{0} .
$$

Before we prove this we will draw its consequences. First of all it implies that for $s>1$ the sum

$$
E\left(v ; \mathbb{H} \backslash B_{s}(\mathrm{i} \eta)\right)+\lim _{\nu \rightarrow \infty} E\left(v^{\nu} ; B_{s}\left(\mathrm{i} y^{\nu} / \delta^{\nu}\right)\right)
$$

is equal to $m_{0}$. Cutting out small neighbourhoods of the bubble points $z_{1}, \ldots, z_{\ell}$ the limit equals the sum of the masses. This shows that

$$
E(v)+m_{1}+\ldots+m_{\ell}=m_{0} .
$$

In particular, i $\eta$ is a bubble point if $v$ is constant. A similar argument and conformal invariance of the energy imply

$$
\lim _{\nu \rightarrow \infty} E\left(w^{\nu}\right)=E(v)+m_{1}+\ldots+m_{\ell}+m_{\infty} .
$$

In order to verify the stability condition observe that

$$
\lim _{\nu \rightarrow \infty} E\left(v^{\nu} ; B_{R}(\mathrm{i} \eta)\right)
$$


is independent of $R>1$ provided that $v$ is constant. Hence,

$$
\lim _{\nu \rightarrow \infty} E\left(v^{\nu} ; B_{R}\left(\mathrm{i} y^{\nu} / \delta^{\nu}\right)\right)=m_{0}
$$

for all $R>1$. Because

$$
E\left(v^{\nu} ; B_{1}\left(\mathrm{i} y^{\nu} / \delta^{\nu}\right)\right)=m_{0}-\hbar / 2
$$

for all $\nu$ and in view of the above cutting argument for the bubble points, which are contained in the closure of $B_{1}(\mathrm{i} \eta)$, there must be a bubble point on the round boundary arc of $B_{1}(\mathrm{i} \eta)$. In other words, $\# Z \geq 2$ provided $v$ is constant.

We prove the above claim, namely that

$$
\lim _{R \rightarrow \infty} \lim _{\nu \rightarrow \infty} E\left(w^{\nu} ; B_{R \delta^{\nu}}\left(z^{\nu}\right)\right)=m_{0}
$$

Observe that for all $R \geq 1$

$$
m_{0}-\hbar / 2 \leq \lim _{\nu \rightarrow \infty} E\left(w^{\nu} ; B_{R \delta^{\nu}}\left(z^{\nu}\right)\right) \leq m_{0} .
$$

Arguing by contradiction we suppose that there exists $\mu>0$ such that for all $R \geq 1$

$$
\lim _{\nu \rightarrow \infty} E\left(w^{\nu} ; B_{R \delta^{\nu}}\left(z^{\nu}\right)\right) \leq m_{0}-\mu
$$

In order to lead this to a contradiction we choose a sequence $\varepsilon^{\nu} \searrow 0$ such that

$$
\lim _{\nu \rightarrow \infty} E\left(w^{\nu} ; B_{\varepsilon^{\nu}}\left(z^{\nu}\right)\right)=m_{0}
$$

see [6, p. 234]. The rescaling argument from [6, p. 234] shows that

$$
\lim _{\nu \rightarrow \infty} E\left(w^{\nu} ; A_{z^{\nu}}\left(\mathrm{e}^{-T} \varepsilon^{\nu}, \varepsilon^{\nu}\right)\right)=0
$$

for all $T>0$. Hence,

$$
m_{0}=\lim _{\nu \rightarrow \infty} E\left(w^{\nu} ; B_{\mathrm{e}^{T} \delta^{\nu}}\left(z^{\nu}\right)\right)+\lim _{\nu \rightarrow \infty} E\left(w^{\nu} ; A_{z^{\nu}}\left(\mathrm{e}^{T} \delta^{\nu}, \mathrm{e}^{-T} \varepsilon^{\nu}\right)\right) .
$$

Moreover, by the definitions of $\delta^{\nu}$ and $\varepsilon^{\nu}$ we have

$$
\lim _{\nu \rightarrow \infty} E\left(w^{\nu} ; A_{z^{\nu}}\left(\delta^{\nu}, \varepsilon^{\nu}\right)\right)=\hbar / 2,
$$

as well as, using the contradiction assumption, that the ratio $\varepsilon^{\nu} / \delta^{\nu}$ tends to infinity. The concentration inequality of Lemma 3.1 implies that

$$
\lim _{\nu \rightarrow \infty} E\left(w^{\nu} ; A_{z^{\nu}}\left(\mathrm{e}^{T} \delta^{\nu}, \mathrm{e}^{-T} \varepsilon^{\nu}\right)\right) \leq c \mathrm{e}^{-T / c} \hbar / 2
$$

for all $T$ sufficiently large. Combining this with the above limit-decomposition of $m_{0}$ we obtain

$$
m_{0} \leq m_{0}-\mu+c \mathrm{e}^{-T / c} \hbar / 2 .
$$

Letting $T$ tend to infinity this yields the desired contradiction.

A similar argumentation to [6. Lemma 3.6] using the distance-energy inequality of Lemma 3.1 shows that the bubbles $w$ and $v$ connect. This finishes the proof of the first case.

For the second we assume that $y^{\nu} / \delta^{\nu}$ tends to infinity. We set

$$
\varphi^{\nu}(z):=x^{\nu}+y^{\nu} z
$$

and obtain a sequence of Möbius transformations preserving the upper half-plane that converges to zero in $C_{\mathrm{loc}}^{\infty}$. We consider the sequence of holomorphic maps obtained by

$$
v^{\nu}:=w^{\nu} \circ \varphi^{\nu}
$$


We claim that $v^{\nu}$ converges modulo bubbling on $\mathbb{H} \backslash$ i to a constant map. Notice, that only i can be a bubble point. Indeed, we have

$$
E\left(v^{\nu} ; B_{\delta^{\nu}} / y^{\nu}(\mathrm{i})\right)=m_{0}-\hbar / 2
$$

for all $\nu$. By the definition of $m_{0}$ we find $\varepsilon>0$ sufficiently small and $\nu$ sufficiently large such that

$$
E\left(w^{\nu} ; B_{\varepsilon}(0)\right) \leq m_{0}+\hbar / 4 .
$$

The term on the left equals the energy of $v^{\nu}$ on the distance disc

$$
B_{\varepsilon / y^{\nu}}\left(-x^{\nu} / y^{\nu}\right)
$$

Hence, for all $\nu$ sufficiently large the energy of $v^{\nu}$ on

$$
D^{\nu}:=B_{\varepsilon / y^{\nu}}\left(-x^{\nu} / y^{\nu}\right) \backslash B_{\delta^{\nu} / y^{\nu}}(\mathrm{i})
$$

is less than or equal to $3 \hbar / 4$. Notice, that the real boundary points $-x^{\nu} / y^{\nu} \pm \varepsilon / y^{\nu}$ of $B_{\varepsilon / y^{\nu}}\left(-x^{\nu} / y^{\nu}\right)$ are of different sign and unbounded as $x^{\nu}$ and $y^{\nu}$ tend to zero. Hence, $D^{\nu}$ approaches all of $\mathbb{H} \backslash$ i. Because the mass of each bubble point is greater or equal to $\hbar$ there is no bubble point on $\mathbb{H} \backslash$ i. Moreover, for all $R>1$ we find $\nu$ such that $A_{\mathrm{i}}(1 / R, R)$ is contained in the domain $D^{\nu}$. Hence, taking the limit for $\nu \rightarrow \infty$ we get

$$
E\left(v ; A_{\mathrm{i}}(1 / R, R)\right) \leq 3 \hbar / 4
$$

for all $R>1$. Taking $R \rightarrow \infty$ we get that $E(v ; \mathbb{H})$ is bounded by $3 \hbar / 4$. The mean value inequality as in Section 2.2 implies that $v$ must be constant. Finally, suppose that $v^{\nu}$ converges in $C_{\text {loc }}^{\infty}$ on the whole closed upper half-plane. Then the energy $E\left(v^{\nu} ; B_{1}(\mathrm{i})\right)$, which is bounded by

$$
E\left(v^{\nu} ; B_{\delta^{\nu}} / y^{\nu}(\mathrm{i})\right)=m_{0}-\hbar / 2
$$

from below, would trend to zero. But this is not possible. Consequently, $v^{\nu}$ converges to a constant map on $\mathbb{H} \backslash \mathrm{i}$ and $\mathrm{i}$ is a bubble point.

Denote the mass of $v^{\nu}$ at $\mathrm{i}$ by $m_{1}$. Because

$$
E\left(v^{\nu} ; B_{\varepsilon}(\mathrm{i})\right) \geq E\left(v^{\nu} ; B_{\delta^{\nu}} / y^{\nu}(\mathrm{i})\right)
$$

for sufficiently large $\nu$ we see taking the limits $\nu \rightarrow \infty$ and $\varepsilon \rightarrow 0$ successively that

$$
m_{1} \geq m_{0}-\hbar / 2 \text {. }
$$

We claim that $m_{0}=m_{1}$ so that no energy gets lost. First of all observe that by the definition of $m_{0}$

$$
\lim _{\varepsilon \rightarrow 0} \lim _{\nu \rightarrow \infty} E\left(w^{\nu} ; B_{\varepsilon}\left(z^{\nu}\right)\right)=m_{0} .
$$

With the decomposition

$$
E\left(v^{\nu} ; B_{\varepsilon}(\mathrm{i})\right)+E\left(v^{\nu} ; A_{\mathrm{i}}\left(\varepsilon, \varepsilon / y^{\nu}\right)\right)
$$

of

$$
E\left(v^{\nu} ; B_{\varepsilon / y^{\nu}}(\mathrm{i})\right)=E\left(w^{\nu} ; B_{\varepsilon}\left(z^{\nu}\right)\right)
$$

this implies that

$$
m_{0}=m_{1}+\lim _{\varepsilon \rightarrow 0} \lim _{\nu \rightarrow \infty} E\left(v^{\nu} ; A_{\mathrm{i}}\left(\varepsilon, \varepsilon / y^{\nu}\right)\right) .
$$


In particular, because $m_{0}-m_{1} \leq \hbar / 2$ the double limes term is bounded by $\hbar / 2$. Hence, there exists $\varepsilon_{0} \in(0,1 / 4)$ such that for all $\varepsilon \in\left(0,2 \varepsilon_{0}\right]$ there exists $\nu_{0} \in \mathbb{N}$ such that for all $\nu \geq \nu_{0}$ we have $4 \leq 1 / y^{\nu}$ and

$$
E\left(v^{\nu} ; A_{\mathrm{i}}\left(\varepsilon, \varepsilon / y^{\nu}\right)\right) \leq 2 \hbar / 3
$$

Notice, that

$$
\lim _{\nu \rightarrow \infty} E\left(v^{\nu} ; A_{\mathrm{i}}(\varepsilon, 1)\right)=0
$$

for all $\varepsilon \in(0,1)$, because $v^{\nu}$ converges uniformly on $A_{\mathrm{i}}(\varepsilon, 1)$ to the constant map. This implies

$$
\lim _{\varepsilon \rightarrow 0} \lim _{\nu \rightarrow \infty} E\left(v^{\nu} ; A_{\mathrm{i}}(\varepsilon, 1)\right)=0 .
$$

In other words it suffices to show that

$$
\lim _{\varepsilon \rightarrow 0} \lim _{\nu \rightarrow \infty} E\left(v^{\nu} ; A_{\mathrm{i}}\left(1, \varepsilon / y^{\nu}\right)\right)=0
$$

In order to do so observe that for all $\varepsilon \in\left(0,2 \varepsilon_{0}\right]$ the annuli $A_{\mathrm{i}}\left(1, \varepsilon / y^{\nu}\right)$ support energy $E\left(v^{\nu} ;.\right) \leq 2 \hbar / 3$ for all $\nu \geq \nu_{0}$ and that the distance discs $B_{r / 2}\left(\mathrm{i}+r \mathrm{e}^{\mathrm{i} \theta}\right)$ are contained in $A_{\mathrm{i}}\left(2 \varepsilon_{0}, 2 \varepsilon_{0} / y^{\nu}\right)$ for all $r \in\left[1, \varepsilon_{0} / y^{\nu}\right]$. Therefore, as in the first part of the proof of the concentration inequality of Lemma 3.1, again invoking the mean value inequality and the conventions about $\hbar$ from Section 2.3, we get

$$
v^{\nu}\left(A_{\mathrm{i}}\left(1, \varepsilon / y^{\nu}\right)\right) \subset \Omega_{L}
$$

for all $\varepsilon \in\left(0, \varepsilon_{0}\right)$, because both boundary components of $A_{\mathrm{i}}\left(1, \varepsilon / y^{\nu}\right)$ intersect the real axis. Hence, we are allowed to work with the symplectic form $\omega_{L}=\mathrm{d} \lambda$ and can proceed as in the second part of the proof of the concentration inequality. Consider the rescaled maps

$$
u^{\nu}:=v^{\nu} \circ \sqrt{\varepsilon / y^{\nu}}
$$

that support energy less than $\hbar$ on

$$
A_{\mathrm{i} \sqrt{y^{\nu} / \varepsilon}}\left(\sqrt{y^{\nu} / \varepsilon}, \sqrt{\varepsilon / y^{\nu}}\right) .
$$

With $\mathrm{e}^{-T}=\sqrt{\varepsilon / \varepsilon_{0}}$ and the concentration inequality we get

$$
E\left(u^{\nu} ; A\left(\mathrm{e}^{T} \sqrt{y^{\nu} / \varepsilon_{0}}, \mathrm{e}^{-T} / \sqrt{y^{\nu} / \varepsilon_{0}}\right)\right) \leq c \mathrm{e}^{-T / c} \hbar .
$$

Notice, that the dependence of the center $\mathrm{e}^{T} \sqrt{y^{\nu} / \varepsilon_{0}}$ of the annulus on $T$ does not affect the inequality because the proof uses logarithmic polar coordinates around $\mathrm{e}^{T} \sqrt{y^{\nu} / \varepsilon_{0}}$. Undoing the rescaling we get that there exists a positive constant $c_{2}$ such that

$$
E\left(v^{\nu} ; A_{\mathrm{i}}\left(1, \varepsilon / y^{\nu}\right)\right) \leq c_{2}(\sqrt{\varepsilon})^{1 / c}
$$

for all $\nu$ sufficiently large. Consequently,

$$
\lim _{\nu \rightarrow \infty} E\left(v^{\nu} ; A_{\mathrm{i}}\left(1, \varepsilon / y^{\nu}\right)\right) \leq c_{2}(\sqrt{\varepsilon})^{1 / c} .
$$

Hence, $m_{0}=m_{1}$ follows letting $\varepsilon$ tend to zero. This finishes the second part because the proof that the bubbles connect is the same as in [6, Lemma 3.6]. This is due to the distance-energy inequality, see Lemma 3.1. The proof is complete.

\section{Generalizations}

In this section we collect some generalizations of Theorem 1.1 . 
4.1. Variation of almost complex structures. The theorem continues to hold if we allow the almost complex structure to vary. One can consider a sequence of $J^{\nu}$-holomorphic discs as well, where $J^{\nu}$ is a sequence of almost complex structures that converges in $C_{\mathrm{loc}}^{\infty}$ to $J$.

4.2. Uniqueness. The limit stable map of a Gromov converging sequence of holomorphic maps is unique up to equivalence. A description can be found in 6, Theorem 3.4]. The images of the Gromov converging holomorphic maps converge in the sense of Hausdorff to the image of the limit stable map, see 6, Proposition $3.2]$ or [16, Theorem A.1].

4.3. Marked stable maps. For bounded sequences of stable maps with bounded energy the notion of Gromov convergence can be defined as in [6, Section 4]. The appearance of marked points and the variation of almost complex structures are allowed. The analog of Theorem 1.1 for marked stable maps of genus zero can be obtained as in [6, Section 4] with the modifications of the present work caused by the totally real boundary condition. In other words, all results from [6] carry over to the situation described in the introduction.

Acknowledgement. We thank Peter Albers, Frédéric Bourgeois, Hansjörg Geiges, and Klaus Niederkrüger who encouraged us to write this article. We thank Urs Fuchs who had explained us his approach to Gromov compactness of his thesis [7].

\section{REFERENCES}

[1] P. Albers, H. Hofer, On the Weinstein conjecture in higher dimensions, Comment. Math. Helv. 84 (2009), 429-436.

[2] F. Bourgeois, A survey of contact homology, in New perspectives and challenges in symplectic field theory, CRM Proc. Lecture Notes 49, Amer. Math. Soc., Providence, RI (2009), $45-71$.

[3] Ya. Eliashberg, Filling by holomorphic discs and its applications, in: Geometry of LowDimensional Manifolds, Vol. 2 (Durham, 1989), London Math. Soc. Lecture Note Ser. 151, Cambridge University Press (1990), 45-67.

[4] Ya. Eliashberg, H. Hofer, A Hamiltonian characterization of the three-ball, Differential Integral Equations 7 (1994), 1303-1324.

[5] Ya. Eliashberg, L. Polterovich, Local Lagrangian 2-knots are trivial, Ann. of Math. (2) 144 (1996), 61-76.

[6] U. Frauenfelder, Gromov convergence of pseudoholomorphic disks, J. Fixed Point Theory Appl. 3 (2008), 215-271.

[7] U. Fuchs, Pseudoholomorphic Curves in Symplectic and Contact Geometry and their Application in Dynamics, Ph.D. Thesis, Purdue University (2014).

[8] H. Geiges, K. Zehmisch, Eliashberg's proof of Cerf's theorem, J. Topol. Anal. 2 (2010), $543-579$.

[9] H. Geiges, K. Zehmisch, How to recognise a 4-ball when you see one, Münster J. Math. 6 (2013), 525-554.

[10] H. Geiges, K. Zehmisch, Reeb dynamics detects odd balls, to appear in: Ann. Sc. Norm. Super. Pisa Cl. Sci. (5), see: arXiv: 1401.3598.

[11] H. Geiges, K. Zenmisch, The Weinstein conjecture for connected sums, to appear in: Int. Math. Res. Not. IMRN, see: arXiv: 1407.4307.

[12] P. Ghiggini, K. Niederkrüger, C. Wende, Subcritical contact surgeries and the topology of symplectic fillings, preprint (2014), arXiv: 1408.1051v2.

[13] M. Gromov, Pseudoholomorphic curves in symplectic manifolds, Invent. Math. 82 (1985), $307-347$.

[14] R. Hind, Filling by holomorphic disks with weakly pseudoconvex boundary conditions, Geom. Funct. Anal. 7 (1997), 462-495. 
[15] H. Hofer, Pseudoholomorphic curves in symplectizations with applications to the Weinstein conjecture in dimension three, Invent. Math. 114 (1993), 515-563.

[16] H. Hofer, D. Salamon, Floer homology and Novikov rings, in: The Floer memorial volume, Progr. Math. 133, Birkhäuser, Basel (1995), 483-524.

[17] H. Hofer, K. Wysocki, E. Zehnder, A characterisation of the tight three-sphere, Duke Math. J. 81 (1995), 159-226.

[18] S. Ivashrovich, V. Shevchishin, Reflection principle and $J$-complex curves with boundary on totally real immersions, Commun. Contemp. Math. 4 (2002), 65-106.

[19] M. Kontsevich, Enumeration of rational curves via torus actions, in: The moduli space of curves (Texel Island, 1994), Progr. Math. 129, Birkhäuser Boston, Boston (1995), 335-368.

[20] P. Massot, K. Niederkrüger, C. Wende, Weak and strong fillability of higher dimensional contact manifolds, Invent. Math. 192 (2013), 287-373.

[21] D. McDuff, D. Salamon, Introduction to symplectic topology, Oxford Mathematical Monographs, Second, The Clarendon Press Oxford University Press, New York, (1998), 1-486.

[22] D. McDuff and D. Salamon, J-holomorphic Curves and Symplectic Topology, Amer. Math. Soc. Colloq. Publ. 52, American Mathematical Society, Providence, RI (2004).

[23] K. NiederkrüGer, The plastikstufe - a generalization of the overtwisted disk to higher dimensions, Algebr. Geom. Topol. 6 (2006), 2473-2508.

[24] K. Niederkrüger, A. Rechtman, The Weinstein conjecture in the presence of submanifolds having a Legendrian foliation, J. Topol. Anal. 3 (2011), 405-421.

[25] J. Sacks, K. Uhlenbeck, The existence of minimal immersions of 2-spheres, Ann. of Math. (2) 113 (1981), 1-24.

[26] R. Ye, Gromov's compactness theorem for pseudo holomorphic curves, Trans. Amer. Math. Soc. 342 (1994), 671-694.

[27] R. Ye, Filling by holomorphic curves in symplectic 4-manifolds, Trans. Amer. Math. Soc. 350 (1998), 213-250.

[28] K. Zenмisch, The Eliashberg-Gromov tightness theorem, Diplomarbeit, Universität Leipzig (2003).

Institut für Mathematik, Universität Augsburg

E-mail address: Urs.Frauenfelder@math.uni-augsburg.de

Mathematisches Institut, Westfälische Wilhelms-Universität MÜNster

E-mail address: Kai.Zehmisch@uni-muenster.de 